\title{
Screening CT Angiography for Pediatric Blunt Cerebrovascular Injury with Emphasis on the Cervical "Seatbelt Sign"
}

\author{
N.K. Desai, J. Kang, and F.H. Chokshi
}

\begin{abstract}
BACKGROUND AND PURPOSE: There are no standard screening guidelines to evaluate blunt cerebrovascular injury in children. The purpose of this retrospective study was to understand the clinical and radiologic risk factors associated with pediatric blunt cerebrovascular injury on CTA of the neck with primary attention to the cervical "seatbelt sign."
\end{abstract}

MATERIALS AND METHODS: Radiology reports from 2002 to 2012 were queried for the examination "CTA neck." The electronic medical record was reviewed for mechanism of injury, Glasgow Coma Scale score, and physical examination findings. Radiology reports from adjunct radiographic studies were reviewed. CTA neck examinations with reported blunt cerebrovascular injury were reviewed to confirm imaging findings. Patients with penetrating injury or those without a history of trauma were excluded.

RESULTS: Four hundred sixty-three patients underwent CTA of the neck; 137 had blunt trauma. Forty-two of 85 patients involved in a motor vehicle collision had a cervical seatbelt sign; none had blunt cerebrovascular injury. Nine vessels (4 vertebral arteries, 4 ICAs, 1 common carotid artery) in 8 patients ultimately were diagnosed with various grades (I-IV) of blunt cerebrovascular injury, representing $5.8 \%(8 / 137)$ of the population screened for blunt neck trauma. The mean Glasgow Coma Scale score was significantly lower $(P=.02)$ in the blunt cerebrovascular injury group versus the non-blunt cerebrovascular injury group. Although not statistically significant, patients with blunt cerebrovascular injury had a higher tendency to have additional traumatic injuries, primarily basilar skull fractures $(P=.05)$ and intracranial hemorrhage $(P=.13)$.

CONCLUSIONS: A common indication for neck CTA, the cervical seatbelt sign, was not associated with blunt cerebrovascular injury. With the exception of Glasgow Coma Scale score, no single risk factor was statistically significant in predicting vascular injury in this series.

ABBREVIATIONS: $\mathrm{BCVI}=$ blunt cerebrovascular injury; $\mathrm{EAST}=$ Eastern Association for the Surgery of Trauma; GCS = Glasgow Coma Scale; MVC = motor vehicle collision; NPTR = National Pediatric Trauma Registry

$T^{1}$ he incidence of blunt cerebrovascular injury (BCVI) in adultpredominant series is estimated at approximately $1 \%$ and as high as $2.7 \%$ in patients with polytrauma. ${ }^{1,2}$ Despite its fairly low incidence, BCVI has potentially catastrophic neurologic sequelae, including serious morbidity with dense neurologic deficits necessitating costly long-term medical care and even death. ${ }^{3}$ Most cur-

Received November 17, 2013; accepted after revision February 1, 2014.

From the Division of Neuroradiology (N.K.D.), Department of Radiology and Imaging Sciences, Emory University School of Medicine, Children's Healthcare of Atlanta, Atlanta, Georgia; Department of Biostatistics and Bioinformatics (J.K.), Department of Radiology and Imaging Sciences, Emory University School of Medicine and Rollins School of Public Health, Atlanta, Georgia; and Division of Neuroradiology (F.H.C.), Department of Radiology and Imaging Sciences, Emory University School of Medicine, Atlanta, Georgia.

Please address correspondence to Nilesh K. Desai, MD, Department of Radiology and Imaging Sciences, Division of Neuroradiology, Emory University School of Medicine, Children's Healthcare of Atlanta, 1364 Clifton Rd. NE, EUH B115, Atlanta, GA 30322; e-mail: nilesh.k.desai@emory.edu

EIndicates article with supplemental on-line table.

http://dx.doi.org/10.3174/ajnr.A3916 rent literature supports fairly liberal screening of adult patients based on these studies, especially with the advent of noninvasive CTA having replaced conventional catheter-based angiography. ${ }^{4,5}$ Although screening criteria for adults seem to be wellestablished, ${ }^{6,7}$ there are currently no standard screening guidelines to evaluate BCVI in children. Moreover, the current recommendations of the Eastern Association for the Surgery of Trauma (EAST), the organization that has published screening guidelines based on the most extensive review of the available literature on this topic, including 68 references from the National Library of Medicine/National Institutes of Health MEDLINE data base, state that pediatric patients should be evaluated by using the same criteria as those used in the adult population. ${ }^{8}$ This recommendation is based on limited case series data, however, for which prospective scientific evidence is lacking. ${ }^{8}$

Two recent retrospective studies have evaluated the applicability of adult criteria set forth by EAST to the pediatric population. ${ }^{9,10}$ While one study supported the notion that risk factors for 


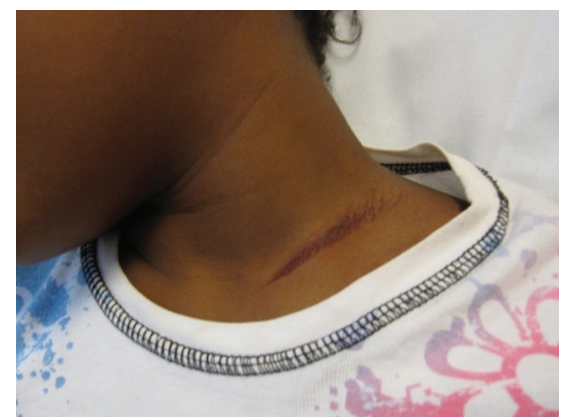

FIG 1. Cervical seatbelt sign. Dermal bruising at the base of the neck, just cephalad to the clavicle indicating injury by a seatbelt. Courtesy of Matthew Clifton, MD, Children's Healthcare of Atlanta, Atlanta, Georgia.

BCVI in children parallel those of adults, the other, in contradistinction, demonstrated that as many as two-thirds of patients experiencing stroke from BCVI did not meet screening criteria according to those used for adults. ${ }^{9,10}$

At our institution, adult criteria seem to be generally followed by our trauma team colleagues. One criterion contained within the criteria proposed for the general population by authors such as Biffl et $\mathrm{al}^{6}$ is that of seatbelt abrasion or seatbelt injury to the anterior neck, the so-called cervical "seatbelt sign" (Fig 1). ${ }^{8}$ While the EAST recommends that the seatbelt sign not be used as an independent criterion without additional risk factors and physical examination findings to stratify patients for screening, ${ }^{8}$ it remains a physical sign that even in isolation, often prompts clinicians to pursue CTA of the neck at our institution and perhaps at many others.

The purpose of this retrospective study was to further understand the risk factors associated with BCVI in children by examining various clinical and radiologic findings on CTA of the neck and adjunct imaging studies, with a primary regard for the predictive value of the cervical seatbelt sign for BCVI.

\section{MATERIALS AND METHODS}

This retrospective study was approved by our local institutional review board and is in compliance with the Health Insurance Portability and Accountability Act. From the PACS at 2 pediatric trauma centers within our institution (a level I and a level II), CTA neck radiology reports between March 2002 and November 2012 were retrieved. The electronic medical record was reviewed for the following: 1) pertinent clinical history; 2) mechanism of injury; 3) Glasgow Coma Scale (GCS) score; and 4) physical examination findings, including the designation by the clinician of cervical seatbelt sign. To consider it a seatbelt injury, the attending pediatric surgeon or pediatric emergency medicine physician must have used terminology explicitly mentioning the presence of seatbelt injury either independently or in cosignature with a trainee note. "Soft-tissue injury of the neck," if so stated, for example, would therefore not qualify as a cervical seatbelt sign for the purpose of this study, even if, in reality, the findings were consistent with a seatbelt injury. Reports from adjunct radiographic, CT, and MR imaging studies performed during the initial trauma encounter were reviewed specifically for internal carotid, common carotid, and vertebral artery injury; intracranial hemorrhage; and fractures of the cervical spine, skull base, maxillofacial region, ribs, and clavicle. Imaging findings of arterial injury were confirmed by independent review of the CTA by 1 of 2 neuroradiologists with Certificates of Added Qualification, (N.K.D. or F.H.C.) for patients with reported BCVIs. In addition, vascular injury severity was graded according to the injury scale proposed by Biffl et $\mathrm{al}^{11}$ : grade I injury indicating intimal irregularity with $<25 \%$ narrowing, grade II injury indicating dissection or intramural hematoma with $>25 \%$ narrowing, grade III indicating pseudoaneurysm, grade IV indicating vessel occlusion, and grade V indicating transection with extravasation. All other findings were recorded on the basis of the radiology report alone. Neuroradiologic studies at our institution are interpreted by Certificate of Added Qualification neuroradiologists. Patients with penetrating injury or without history of trauma were excluded.

Neck CTA was performed on 1 of 2 CT scanners: between March 2002 and May 2006, each CTA was performed on a 16-slice unit (Lightspeed VCT; GE Healthcare, Milwaukee, Wisconsin); this was subsequently replaced with a 64-slice unit (Lightspeed VCT; GE Healthcare) in June 2006. Standard helical CTA neckscanning protocol was used from the aortic arch to the cranial margin of the sella turcica with the technique tailored to each patient with $120 \mathrm{kV}$ (peak) for all patients and a variable manual technique based on weight with predesignated milliampere-second ranging from 200 to 500 and adaptive statistical iterative reconstruction at $10 \%$. Table speed ranged from 19.37 to 39.37 $\mathrm{mm} / \mathrm{s}$ with a rotation time of 0.4 seconds and a pitch at 0.984 . Images were acquired at $0.625-\mathrm{mm}$ section thickness in soft-tissue algorithm. Injection rates and technique, either via central or peripheral intravenous lines, would have varied with age and the caliber of the venous line, with the youngest of children below 15 $\mathrm{kg}$ requiring manual injection and all others requiring power injection at 3-4 mL/s (Medrad, Pittsburgh, Pennsylvania). Our policy for CTA is that the patient either already has or that all reasonable attempts are made to establish a 20-ga or larger intravenous line, especially for older children. Power injection is only performed in patients with at least a 22 -ga intravenous line. For children under $20 \mathrm{~kg}$, we typically perform a timing delay based on data we have collected at our institution. This includes an 8- to 9-second delay for patients up to $14 \mathrm{~kg}$ and a 9- to 10-second delay for patients $15-19 \mathrm{~kg}$. In children $\geq 20 \mathrm{~kg}$, a monitoring scan was used, with a small test bolus to calculate the delay time for the actual examination. Optiray 320 (ioversol; Mallinckrodt, St. Louis, Missouri) was administered at a dosage of $2.0 \mathrm{~mL} / \mathrm{kg}$ with a maximum dosage of $150 \mathrm{~mL}$ for all patients.

\section{Statistical Analysis}

Contingency tables were created for the presence of BCVI by the CTA and each of the other examinations (CT of the cervical spine, which was performed by default in all patients as a result of the CTA neck coverage, cervical spine radiographs, noncontrast head CT, maxillofacial CT, chest radiographs, and chest CT). Sensitivity, specificity, positive predictive value, and negative predictive value were calculated for the outcome of the CTA and the presence of cervical spine, basilar skull, maxillofacial, rib, and clavicle fractures and intracranial hemorrhage. Descriptive statistics (mean, SD, and frequency) were summarized for GCS score and 


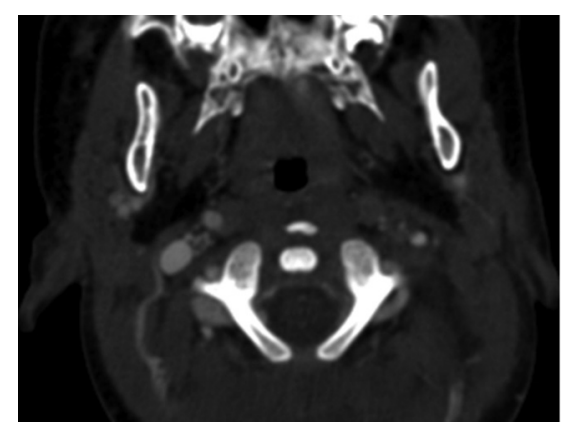

FIG 2. Internal carotid artery injury in patient 6. CTA of the neck demonstrating occlusion of the left internal carotid artery (grade IV injury) at the level of $\mathrm{Cl}$ vertebral body in a 1.17-year-old boy who presented neurologically intact after experiencing a witnessed lowspeed motor-pedestrian collision.

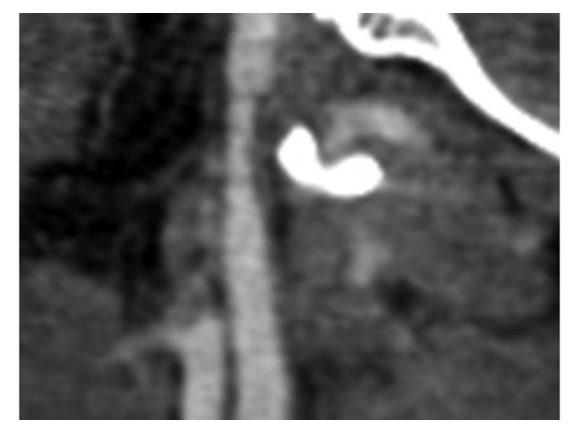

FIG 3. Internal carotid artery injury in patient 3 . Sagittal reformat of a neck CTA image showing $>25 \%$ ICA narrowing related to grade II injury in a 9.92-year-old girl, status post MVC with ejection, who was found unconscious in the field with fixed and dilated pupils.

demographic variables. Also, a Fisher exact test was used to check the independence between the CTA and each of the other examinations. A Wilcox test was used to compare GCS scores between BCVI and non-BCVI groups. A logistic regression model by using age to predict BCVI or lack of BCVI was performed.

\section{RESULTS}

During the nearly 10-year period, 463 patients underwent CTA of the neck for various indications, of whom, 152 had blunt neck trauma. Fifteen patients were excluded due to incomplete medical charts and documentation of mechanism of injury or because a physical examination could not be obtained. Incomplete medical charts were, in all cases, physician notes that had failed to become available during the transition from paper to electronic medical records, primarily between 2002 and 2004. The average age of patients was $9.60 \pm 4.32$ years, including 74 male and 63 female patients ranging between 0.67 and 17 years of age, with 18 patients younger than 5 years. The average age of patients involved in a motor vehicle collision (MVC) $(n=85)$ was $9.5 \pm 4.39$ years, ranging from 1.33 to 17 years of age.

Nine vessels (4 vertebral arteries, 4 ICAs, 1 common carotid artery) in 8 patients ultimately were diagnosed with various grades (I-IV) of BCVI, representing 5.8\% (8/137) of the blunt neck trauma population (On-line Table and Figs 2 and 3). The mean age of patients with BCVI was $8.19 \pm 6.14$ years, ranging from 1.17 to 16 years of age, whereas those without BCVI had a mean age of $9.65 \pm 4.20$ years, ranging from 0.67 to 17 years of age.
Five patients with vascular injury were not involved in an MVC (On-line Table).

All patients with BCVI had at least 1 nonvascular injury such as cervical spine fracture (Table 1). Nearly statistically significant, patients with BCVI had a higher tendency to have additional traumatic injuries, primarily basilar skull fractures $(P=.05)$ and intracranial hemorrhage $(P=.13)$ (Table 2$)$. Of the 4 patients with vertebral artery injury, $2 / 4$ had cervical spine fracture, whereas both cervical spine fractures and vertebral artery injuries were found in 2 additional patients. Additionally, 6 of 8 patients with BCVI and 107 of 129 without BCVI had documented GCS scores. In patients with BCVI, the mean GCS score was $8.67 \pm 6.22$ versus $12.92 \pm 3.90$ in patients without BCVI. This difference was statistically significant $(P=.02)$. Age was not a statistically significant predictive factor for BCVI or a lack of BCVI (logistic regression coefficient $=-.04, P=.64$ ).

Of the patients with BCVI, 3 had neurologic sequelae. One patient, patient 3, an unrestrained ejected motor vehicle passenger, developed cerebral infarction (total MCA distribution) with eventual death secondary to severe closed-head injury with malignant cerebral edema and multicompartmental hemorrhage resulting in transtentorial herniation. Large MCA distribution infarct may have been contributory but not primary to this patient's death (On-line Table). Patient 4 presented with right hemiparesis with MR imaging-confirmed foci of an embolic-type infarction in the left frontal and temporal lobes after an MVC. Another patient, patient 8 , presented with head and neck pain, tingling in the hands, facial paresthesias, and subjective oral motor difficulty several days after a dirt bike accident (On-line Table).

Of eighty-five patients involved in an MVC, 42 had a documented cervical seatbelt sign with an average age of $8.5 \pm 3.84$ years, none of whom had BCVI (positive predictive value $=0$ ). None of the 3 patients with BCVI involved in an MVC had a documented cervical seatbelt sign. Twenty-two additional patients in an MVC had some form of soft-tissue injury to the neck that was not specifically listed as a seatbelt sign. Of these 22 patients, patient 7 demonstrated BCVI with a grade IV right cervical vertebral artery injury. This patient, who later died from severe cerebral edema and hemorrhage with probable upper cervical cord transection, met multiple criteria (EAST criteria) for screening, including a GCS score of 3 and a C4-C5 fracture dislocation injury.

\section{DISCUSSION}

The Eastern Association for the Surgery of Trauma has put forth recommendations for screening criteria for BCVI in the adult population based on the most extensive review of references on this topic. ${ }^{8}$ The EAST recommends screening for BCVI in adult patients with any neurologic abnormality that is not explained by a diagnosed traumatic injury and in trauma patients with epistaxis from a suspected arterial source. For asymptomatic, adult blunt trauma patients with the following risk factors screening is also recommended: Glasgow Coma Scale (GCS) score $\leq 8$; petrous temporal bone fracture, diffuse axonal injury (DAI), cervical spine fracture especially those from $\mathrm{C} 1$ to $\mathrm{C} 3$, fracture through the foramen transversarium, and those with a rotational component or subluxation and Le Fort II or III facial fractures. While the 
Table 1: Associated injuries in patients with and without blunt cerebrovascular injury

\begin{tabular}{lrrrrrrrrrrrr}
\hline & \multicolumn{2}{c}{$\begin{array}{c}\text { Cervical Spine } \\
\text { Fracture }\end{array}$} & \multicolumn{2}{c}{$\begin{array}{c}\text { Basilar Skull } \\
\text { Fracture }\end{array}$} & \multicolumn{2}{c}{$\begin{array}{c}\text { Intracranial } \\
\text { Hemorrhage }\end{array}$} & \multicolumn{2}{c}{$\begin{array}{c}\text { Maxillofacial } \\
\text { Fracture }\end{array}$} & \multicolumn{2}{c}{$\begin{array}{c}\text { Clavicle } \\
\text { Rib Fracture }\end{array}$} \\
\hline BCVI & No & Yes & No & Yes & No & Yes & No & Yes & No & Yes & No & Yes \\
No & 108 & 21 & 79 & 24 & 55 & 27 & 9 & 20 & 84 & 11 & 89 & 6 \\
Yes & 6 & 2 & 4 & 4 & 3 & 5 & 1 & 2 & 6 & 1 & 6 & 1 \\
$\begin{array}{l}\text { Percentage } \\
\text { No }\end{array}$ & 83.7 & 16.2 & 76.7 & 23.3 & 67.1 & 32.9 & 31.0 & 69.0 & 88.4 & 11.6 & 88.4 & 11.6 \\
Yes & 75.0 & 25.0 & 50.0 & 50.0 & 37.5 & 62.5 & 33.3 & 66.7 & 85.7 & 14.3 & 85.7 & 14.3 \\
\hline
\end{tabular}

Table 2: Risk factor analysis for blunt cerebrovascular injury based on associated injuries

\begin{tabular}{lllccc}
\hline & PPV & NPV & Sensitivity & Specificity & P Value \\
\hline Cervical spine fracture & 0.09 & 0.95 & 0.25 & 0.84 & .62 \\
Basilar skull fracture & 0.14 & 0.96 & 0.50 & 0.81 & .05 \\
Intracranial hemorrhage & 0.16 & 0.95 & 0.63 & 0.67 & .13 \\
Maxillofacial fracture & 0.09 & 0.9 & 0.67 & 0.31 & 1 \\
Rib fracture & 0.08 & 0.93 & 0.14 & 0.88 & 1 \\
Clavicle fracture & 0.14 & 0.94 & 0.14 & 0.94 & .4 \\
\hline
\end{tabular}

Note:-PPV indicates positive predictive value; NPV, negative predictive value.

EAST practice guidelines offer a level III recommendation that pediatric patients be screened by using these same adult criteria, prospective trials evaluating risk factors and therefore screening criteria for children are lacking in the current literature. ${ }^{8}$

Furthermore, the EAST recommends that an isolated cervical seatbelt sign should not be used as a screening reason without other risk factors in the presence of normal physical examination findings; however, the cervical seatbelt sign has been endorsed in retrospective series, including Biffl et al, ${ }^{6}$ as a single criterion for screening for BCVI. ${ }^{8}$ One retrospective review by Rozycki et $\mathrm{al}^{12}$ addressed the possible clinical relevance of the cervical seatbelt sign. In 131 patients with seatbelt sign, 4 patients (3\% of the screened population) were found to have carotid artery injuries using CTA or conventional angiography with the presence of BCVI strongly associated with a GCS score of $<14$, Injury Severity Score $>16$, and clavicle and/or first rib fracture in a statistically significant manner. ${ }^{12}$ While the authors in this study concluded that the cervicothoracic seatbelt sign and abnormal neurovascular physical examination findings are an effective combination in screening for BCVI for both adult and pediatric patients, ${ }^{12}$ we believe that there is insufficient evidence based on the small number of pediatric patients $(n=16)$ and the relatively mild or even absent additional injuries to definitively recommend such criteria in children. ${ }^{12}$ Most interesting, on the basis of the available information in the series by Rozycki et al, the EAST guidelines would not have called for screening in 3 of 4 patients with seatbelt signs and BCVI. Therefore, the recommendation by the EAST regarding the cervical seatbelt sign warrants further investigation in both adults and children.

Our study was performed to address the unresolved issues regarding BCVI screening related to cervical seatbelt injury in children; this criterion is still used at our 2 children's hospitals. Of the 85 children in an MVC, 42 demonstrated a cervical seatbelt injury, but none had BCVI. While there was a higher tendency for patients with BCVI to have polytrauma including basilar skull fracture and intracranial hemorrhage, no single risk factor for BCVI met statistical significance in our series with the exception of GCS score (Table 2). Although GCS score $\leq 8$ is used as part of the adult criteria for BCVI screening, our study may offer some initial information about the importance of the GCS score as a screening marker in the pediatric population.

The incidence of BCVI in children is rare. We found that despite nearly 150 patients undergoing CTA screening for blunt injury, the incidence of BCVI was unsurprisingly small, though we did not calculate an incidence due to the unavailability of the number of admissions for blunt trauma to the emergency department. In a recent retrospective case series of nearly 15,000 patients from the general population by Jones et $\mathrm{al}^{9}$ during a 15 -year period, only 45 patients were diagnosed by either CTA or conventional angiography with BCVI, representing $0.3 \%$ of the admitted population. ${ }^{13}$ Another similar recent pediatric retrospective study by Kopelman et $\mathrm{al}^{10}$ demonstrated an incidence of $0.9 \%$. Last, in a review of the National Pediatric Trauma Registry (NPTR) in a more remote study between December 1987 and July 1997 , only 15 of 57,659 registered patients with blunt trauma were diagnosed with BCVI, representing an incidence of $0.03 \% .{ }^{13}$ Admittedly, the relative increase in the incidence found in more recent reviews compared with the NPTR is likely due to increasing physician awareness and use of noninvasive CTA screening.

While it was not our primary goal to directly investigate or propose complete screening criteria for the pediatric population, it is clear that most patients with BCVI in our series met screening criteria as suggested by the EAST recommendations, with the exception of patients 5,6 , and 8 , representing a not insignificant $37.5 \%$ of the BCVI population. Patient 6 experienced a motorpedestrian collision with skull base fractures, including temporal bone fractures but without involvement of the petrous portion. Two patients did have evidence of high-force thoracic injuries not only by history (all-terrain vehicle and dirt bike crash) but also by coincident injury as evidenced by first-rib fracture in patient 5 and T4 compression fracture in patient 8 , which the EAST recommendations do recognize, along with severe head injury, as pediatric risk factors for BCVI, given the findings from the NPTR. ${ }^{8,13}$ Our results are, thus, generally in agreement with that of Jones et $\mathrm{al}^{9}{ }^{9}$ in that adult criteria may not direct clinicians to screen for BCVI in all cases. The inclusion of mechanism, specifically those involving high forces, and significant thoracic injuries may improve such criteria for children. For those patients without BCVI, 89 patients did not meet the EAST screening criteria, whereas 40 did.

There are inherent limitations to our study, many of which originate from the retrospective nature of our series. The primary objective being evaluated, the cervical seatbelt sign, was deemed present on the basis of the clinician specifically recording the exact term in the medical chart. Therefore, while a patient may have had a true cervical seatbelt sign, if the physician only recorded "neck abrasion" or "bruising of the neck," such a patient would not be 
deemed to have the sign by our criteria. While we admit that there may have been a subset of patients missed, we considered it important also to ensure that those who were included as having such a clinical sign were with certainty truly positive. Patient 7 , in fact, did have recorded "anterior neck swelling" without a recorded seatbelt injury. However, this child was noted to have a GCS score of 3 and severe cervical spine injury, including fracture dislocation at $\mathrm{C} 4-\mathrm{C} 5$ with severe distraction, findings that would warrant screening for BCVI. Another uncorrectable issue is the lack of standardization and agreed-upon clinical findings that qualify as a seatbelt injury. It is conceivable that a light abrasion on the low neck may be entirely dismissed by one clinician, but yet another may have recorded the finding as a cervical seatbelt sign. Further, it is possible that recording such a finding may have been forgotten altogether or not properly assessed due to a variety of factors.

In this series, we chose to include patients by searching a radiology data base of all CTA neck examinations performed since early 2002 followed by an electronic medical chart search for all other information. It is, therefore, possible and certainly likely that a child with a cervical seatbelt injury may have not received a screening CTA, depending on physician judgment and lack or presence of coexisting injuries. While we would assume that this patient population would ultimately not be diagnosed with BCVI, this study did not attempt to evaluate all children presenting to the emergency department with a cervical seatbelt sign but rather investigated a CTA-screened population. Finally, we purposely chose to evaluate children with CTA because this remains the standard for noninvasive screening of adults and children at our institution. For $>10$ years, since the presence of our 16-slice and now 64-slice CT scanners, CTA has entirely replaced conventional angiography for BCVI screening. We believe it is possible, however, that a small number of patients very early in our retrospective series may have undergone digital subtraction angiography for possible BCVI.

And finally, our series did not include analysis of the presence or type of restraint because such data were often not recorded in the medical records. Car seats and booster seats generally prevent cervical seatbelt injuries by improving the position of the shoulder strap from over the neck to over the shoulder. The seatbelt sign, therefore, should occur less frequently in young patients who are properly restrained in such devices. In fact, car seats with 5-point harnesses seldom cause seatbelt injury because the straps do not cross over the cervicothoracic region. We believe that restraint history and protective effects should be considered in future prospective trials assessing pediatric risk factors for BCVI.

\section{CONCLUSIONS}

BCVI is an uncommon occurrence in the pediatric trauma population. A common indication for neck CTA, the cervical seatbelt sign, was not associated with BCVI. These findings suggest that especially when found in isolation, the seatbelt sign in children may not be an appropriate reason to perform neck CTA, consistent with EAST recommendations. With the exception of GCS score, no single risk factor was statistically significant in predicting vascular injury in this series, though there was a tendency for those with vascular injury to have multiple risk factors, especially basilar skull fractures and intracranial hemorrhage. Larger, multicenter prospective trials evaluating BCVI in children should be undertaken.

Disclosures: Nilesh K. Desai-UNRELATED: Grants/Grants Pending: Siemens USA, * Comments: DTI metrics using DTI Resolve in patients with cervical myelopathy secondary to spondylosis. Role of coinvestigator with $1 \%$ salary support. *Money paid to the institution.

\section{REFERENCES}

1. Biffl WL, Moore EE, Ryu RK, et al. The unrecognized epidemic of blunt carotid arterial injuries: early diagnosis improves neurologic outcome. Ann Surg 1998;228:462-70

2. Mutze S, Rademacher G, Matthes G, et al. Blunt cerebrovascular injury in patients with blunt multiple trauma: diagnostic accuracy of duplex Doppler US and early CT angiography. Radiology 2005;237:884-92

3. Cothren CC, Moore EE, Ray CE Jr, et al. Screening for blunt cerebrovascular injuries is cost-effective. Am J Surg 2005;190:845-49

4. Chokshi FH, Munera F, Rivas LA, et al. 64-MDCT angiography of blunt vascular injuries of the neck. AJR Am J Roentgenol 2011;196:W309-15

5. Munera F, Foley M, Chokshi FH. Multi-detector row CT angiography of the neck in blunt trauma. Radiol Clin North Am 2012;50:59-72

6. Biffl WL, Moore EE, Offner PJ, et al. Optimizing screening for blunt cerebrovascular injuries. Am J Surg 1999;178:517-22

7. Cothren CC, Moore EE, Biffl WL, et al. Anticoagulation is the gold standard therapy for blunt carotid injuries to reduce stroke rate. Arch Surg 2004;139:540-45, discussion 545-46

8. Bromberg WJ, Collier BC, Diebel LN, et al. Blunt cerebrovascular injury practice management guidelines: the Eastern Association for the Surgery of Trauma. J Trauma 2010;68:471-77

9. Jones TS, Berlew CC, Kornblith LC, et al. Blunt cerebrovascular injuries in the child. Am J Surg 2012;204:7-10

10. Kopelman TR, Berardoni NE, O’Neill PJ, et al. Risk factors for blunt cerebrovascular injury in children: do they mimic those seen in adults? J Trauma 2011;71:559-64, discussion 564

11. Biffl WL, Moore EE, Offner PJ, et al. Blunt carotid arterial injuries: implications of a new grading scale. J Trauma 1999;47:845-53

12. Rozycki GS, Tremblay L, Feliciano DV, et al. A prospective study for the detection of vascular injury in adult and pediatric patients with cervicothoracic seat belt signs. J Trauma 2002;52:618-23, discussion 623-24

13. Lew SM, Frumiento C, Wald SL. Pediatric blunt carotid injury: a review of the National Pediatric Trauma Registry. Pediatr Neurosurg 1999;30:239-44 